\title{
Learning Classifiers without Negative Examples: A Reduction Approach
}

\author{
Dell Zhang \\ SCSIS \\ Birkbeck, University of London \\ London WC1E 7HX, UK \\ dell.z@ieee.org
}

\author{
Wee Sun Lee \\ Department of Computer Science \\ National University of Singapore \\ Singapore 117590 \\ leews@comp.nus.edu.sg
}

\begin{abstract}
The problem of $P U$ Learning, i.e., learning classifiers with positive and unlabelled examples (but not negative examples), is very important in information retrieval and data mining. We address this problem through a novel approach: reducing it to the problem of learning classifiers for some meaningful multivariate performance measures. In particular, we show how a powerful machine learning algorithm, Support Vector Machine, can be adapted to solve this problem. The effectiveness and efficiency of the proposed approach have been confirmed by our experiments on three real-world datasets.
\end{abstract}

\section{Introduction}

Standard machine learning techniques for building a binary classifier require a set of positive examples $\mathbf{x}$ with label +1 and a set of negative examples $\mathbf{x}$ with label -1 . However, in practice it is often very difficult to get labelled negative examples. This is because people usually only keep the data that are of interest to them (i.e., positive examples), and it is unnatural to require people to label uninteresting data (i.e. negative examples). In such situations, what available in addition to positive examples is just a set of unlabelled examples. Can we still train classifiers effectively and efficiently without any negative examples? This problem is called PU Learning [9].

Definition 1. PU Learning. Given an incomplete set of positive examples $P=\left\{\mathbf{x}_{1}, \ldots, \mathbf{x}_{l}\right\}$ that we are interested in, and a set of unlabelled examples $U=\left\{\mathbf{x}_{l+1}, \ldots, \mathbf{x}_{l+u}\right\}$ which contains both positive examples and negative examples, we would like to use $P$ and $U$ to train a classifier that can accurately classify positive and negative examples in $U$ or in a separate test set.

The problem of PU Learning occurs frequently in information retrieval and data mining applications [9]. For ex- ample, a researcher may have saved in her computer some journal articles on a specialised subtopic in bioinformatics $(P)$, and she wants to find more materials on that subtopic from the PubMed Central digital library $(U)$. For another example, a user searched the Web using a search engine and clicked on some returned links that he was interested in, then the search engine could improve its ranking of the search results by building a classifier based on the clicked links $(P)$ and the other links $(U)$.

We address this problem through a novel approach: reducing it to the problem of learning classifiers for some meaningful multivariate performance measures.

One of the most powerful machine learning techniques for classification is Support Vector Machine (SVM) [13] which has solid theoretical basis and broad practical success. For example, SVM in its simplest form, linear SVM, consistently provides state-of-the-art performance for text categorization tasks [16]. In this paper, we focus on adapting SVM algorithms for PU Learning, though our reduction approach to PU Learning is general.

The rest of this paper is organised as follows. We first propose our reduction approach to PU Learning (in Section 2), then present experimental evaluation (in Section 3), later discuss related work (in Section 4), and finally make conclusions (in Section 5).

\section{Approach}

\subsection{Learning from $P$ and $N$}

In the ideal situation, we have in addition to $P$ a set of negative examples $N$ rather than a set of unlabelled examples $\left(\forall \mathbf{x}_{i} \in P: y_{i}=+1\right.$ and $\forall \mathbf{x}_{i} \in N: y_{i}=-1$ ), so we can use both $P$ and $N$ to train a standard SVM classifier.

OP 1. $\mathrm{SVM}_{2 C}$

$$
\min _{\mathbf{w}, \xi_{i} \geq 0} \frac{1}{2} \mathbf{w}^{T} \mathbf{w}+\frac{C}{n} \sum_{i=1}^{n} \xi_{i}
$$




$$
\text { s.t. } \quad \forall_{i=1}^{n}: y_{i} \mathbf{w}^{T} \mathbf{x}_{i} \geq 1-\xi_{i}
$$

$\mathrm{SVM}_{2 C}$ attempts to find a hyperplane $h(\mathbf{x})=\mathbf{w}^{T} \mathbf{x}$ that can separate positive examples and negative examples with a large margin $\frac{2}{\|\mathbf{w}\|}$ as well as small empirical hinge loss $\sum_{i=1}^{n} \xi_{i}$.

However, as explained earlier, a negative set $N$ is often not available therefore $\mathrm{SVM}_{2 C}$ is not applicable.

\subsection{Learning from $P$ Only}

When we do not have negative examples, one possibility is to ignore $U$ and use $P$ only to train the so-called 'oneclass' SVM classifier [13].

OP 2. $\mathrm{SVM}_{1 c}$

$$
\begin{aligned}
\min _{\mathbf{w}, \xi_{i} \geq 0} & \frac{1}{2} \mathbf{w}^{T} \mathbf{w}+\frac{C}{l} \sum_{i=1}^{l} \xi_{i} \\
\text { s.t. } & \forall_{i=1}^{l}: \mathbf{w}^{T} \mathbf{x}_{i} \geq 1-\xi_{i}
\end{aligned}
$$

Note that our $\mathrm{SVM}_{1 c}$ formulation here is different with the original one-class SVM [13] which relies on a parameter $\nu$ (as in $\nu$-SVM [13]) but not $C$. The parameter $\nu$ has more intuitive sense than $C$ : it directly controls the amount of margin errors and the number of support vectors. Nevertheless, the classification function of the original one-class SVM optimised for a given $\nu$ would be same as that of $\mathrm{SVM}_{1 c}$ with a certain value of $C$. Current training methods for $\nu$-SVMs (including the original one-class SVM) take at least quadratic time, whereas with our $\mathrm{SVM}_{1 c}$ formulation we can achieve linear-time training [20].

Intuitively, $\mathrm{SVM}_{1 c}$ is inferior for PU Learning because it ignores useful information that is present in the set of unlabelled examples $U$.

\subsection{Learning from $P$ and $U$}

If we take the positive examples in $U$ as noise, then we can consider $U$ as a very noisy set of negative training examples. There are totally $n=l+u$ examples. Denote the observed label of an example $\mathbf{x}$ by $y \in\{-1,+1\}$, i.e., $\forall \mathbf{x}_{i} \in P: y_{i}=1$ and $\forall \mathbf{x}_{i} \in U: y_{i}=-1$. Denote the actual label of an example $\mathbf{x}$ by $z \in\{-1,+1\}$ indicating its true relevancy. We know that $\forall \mathbf{x}_{i} \in P: z_{i}=y_{i}=1$, but we have no idea about the hidden value of $z_{i}$ for any $\mathbf{x}_{i} \in U$.

Assume that the examples in $P$ are randomly sampled from the class of positive examples with a certain probability $\mu$. The value of $\mu=\operatorname{Pr}[y=1 \mid z=1]$ is an unknown constant. In other words, an actual positive example has probability $\mu$ to be observed in $P$ and probability $1-\mu$ to be left in $U$; while all actual irrelevant documents are put in $U$. We have the following relationships:

$$
\begin{aligned}
& \operatorname{Pr}[y=+1]=\operatorname{Pr}[z=+1] \mu \\
& \operatorname{Pr}[y=-1]=\operatorname{Pr}[z=-1]+\operatorname{Pr}[z=+1](1-\mu) .
\end{aligned}
$$

Using $P$ and $U$ straightforwardly to train the standard $\mathrm{SVM}_{2 C}$ would not work. Let's call the classification performance calculated over observed (noisy) labels $y_{i} o b$ served performance, and the classification performance calculated over actual labels $z_{i}$ actual performance. SVM $2 C$ minimises the observed error rate, but low observed error rate does not necessarily lead to low actual error rate [1]. For example, when there are 100 documents in $U$ relevant to the given query $P$ that consists of 10 documents, the actual optimal classifier $h_{1}$ would generate 100 observed errors, in contrast, the classifier $h_{2}$ which classifies all examples to be negative would generate 10 observed errors, consequently $h_{2}$ is favoured by $\mathrm{SVM}_{2 C}$ over the actual optimal classifier $h_{1}$.

Our key insight is that we are able to train classifiers in the PU Learning setting, if we substitute some other multivariate performance measures for error rate.

Joachims has proposed a SVM formulation that directly minimises the loss function $\Delta$ corresponding to a multivariate performance measure [5].

$$
\begin{array}{ll}
\text { OP 3. } & \text { SVM }^{\text {erf }} \\
\min _{\mathbf{w}, \xi \geq 0} & \frac{1}{2} \mathbf{w}^{T} \mathbf{w}+C \xi \\
\text { s.t. } & \forall \bar{y}^{\prime} \in\{+1,-1\}^{n} \backslash \bar{y}: \\
& \frac{1}{2 n} \mathbf{w}^{T} \sum_{i=1}^{n}\left(y_{i}-y_{i}{ }^{\prime}\right) \mathbf{x}_{i} \geq \frac{1}{2 n} \Delta\left(\bar{y}^{\prime}, \bar{y}\right)-\xi
\end{array}
$$

Note that our SVM ${ }^{\text {perf }}$ formulation here has a slight difference with its original version [5]: a constant factor $\frac{1}{2 n}$ is introduced to the constraints in order to better capture how $C$ scales with training set size [6].

\section{Balanced Accuracy}

The balanced accuracy of a classifier is the arithmetic average of sensitivity and specificity [14]. It is also known as the Area Under the ROC Curve (AUC) for just one run [14].

The actual balanced accuracy is

$$
B=\frac{\operatorname{Pr}[h(\mathbf{x})=1 \mid z=1]+\operatorname{Pr}[h(\mathbf{x})=-1 \mid z=-1]}{2} .
$$

The observed balanced accuracy is

$$
\widehat{B}=\frac{\operatorname{Pr}[h(\mathbf{x})=1 \mid y=1]+\operatorname{Pr}[h(\mathbf{x})=-1 \mid y=-1]}{2} .
$$


Theorem 1. $\widehat{B}-\frac{1}{2} \propto B-\frac{1}{2}$.

Proof. It can be shown with some simple calculation that [1]

$$
\begin{aligned}
& (2 \widehat{B}-1) \operatorname{Pr}[y=1] \operatorname{Pr}[y=-1] \\
= & (2 B-1) \operatorname{Pr}[z=1] \operatorname{Pr}[z=-1] \mu
\end{aligned}
$$

Therefore, we have

$$
\begin{aligned}
\widehat{B}-\frac{1}{2} & =\left(B-\frac{1}{2}\right) \mu \frac{\operatorname{Pr}[z=1] \operatorname{Pr}[z=-1]}{\operatorname{Pr}[y=1] \operatorname{Pr}[y=-1]} \\
& \propto B-\frac{1}{2}
\end{aligned}
$$

This theorem implies that we can optimise the actual balanced accuracy $B$ by optimising the observed balanced accuracy $\widehat{B}$.

Since $0 \leq \widehat{B} \leq 1$, we define the corresponding multivariate loss function as

$$
\Delta_{b a}(\bar{h}(\overline{\mathbf{x}}), \bar{y})=1-\widehat{B} .
$$

Let $\mathrm{SVM}_{b a}^{\text {perf }}$ denote the $\mathrm{SVM}^{\text {perf }}$ with the loss function $\Delta_{b a}$.

We are able to train $\mathrm{SVM}_{b a}^{\text {perf }}$ efficiently by transforming it to a specific case of SVM ${ }^{\text {struct }}$ - the structural SVM formulation which was first proposed for training SVMs to predict structural outputs [6]. For this purpose we have extended the original $\mathrm{SVM}^{\text {struct }}$ [6] to assign different weights $\lambda_{i}$ to errors on different training examples $\mathbf{x}_{i}$.

\section{OP 4. $\mathrm{SVM}_{b a}^{\text {struct }}$}

$$
\begin{aligned}
\min _{\mathbf{w}, \xi \geq 0} & \frac{1}{2} \mathbf{w}^{T} \mathbf{w}+C \xi \\
\text { s.t. } & \forall \bar{\eta} \in\{0,1\}^{n} \backslash \overline{0}: \\
& \frac{1}{n} \mathbf{w}^{T} \sum_{i=1}^{n} \eta_{i} y_{i} \mathbf{x}_{i} \geq \frac{1}{n} \sum_{i=1}^{n} \eta_{i} \lambda_{i}-\xi \\
& \lambda_{i}=1 /(4 l) \text { if } y_{i}>0 \text { and } \lambda_{i}=1 /(4 u) \text { if } y_{i}<0
\end{aligned}
$$

Theorem 2. $\mathrm{SVM}_{b a}^{\text {perf }}$ is equivalent to $\mathrm{SVM}_{b a}^{\text {struct }}$.

Proof. $\mathrm{SVM}_{b a}^{\text {perf }}$ and $\mathrm{SVM}_{b a}^{\text {struct }}$ have the same objective function to optimise, so we only need to show that they have an equivalent set of constraints.

For each $\bar{y}^{\prime} \in\{+1,-1\}^{n} \backslash \bar{y}$, there is a unique corresponding $\bar{\eta} \in\{0,1\}^{n} \backslash \overline{0}$ through the following one-to-one map:

$$
\eta_{i}=\left\{\begin{array}{ll}
0 & \text { if } y_{i}{ }^{\prime}=y_{i} \\
1 & \text { if } y_{i}{ }^{\prime} \neq y_{i}
\end{array} .\right.
$$

So $\left(y_{i}-y_{i}{ }^{\prime}\right) / 2=\eta_{i} y_{i}$, and the left-hand-expression of each inequality constraint in $\mathrm{SVM}_{b a}^{\text {perf }}$ is same as that in
$\mathrm{SVM}_{b a}^{\text {struct }}$. Now let's look at the right-hand-expression of each inequality constraint. Noticing $c=\sum_{y_{i}>0} \eta_{i}$ and $d=$ $\sum_{y_{i}<0} \eta_{i}$, we can re-write $\sum_{i=1}^{n} \eta_{i} \lambda_{i}$ as

$$
\begin{aligned}
\sum_{y_{i}>0} \eta_{i} \lambda_{i}+\sum_{y_{i}<0} \eta_{i} \lambda_{i} & =\frac{1}{4 l} \sum_{y_{i}>0} \eta_{i}+\frac{1}{4 u} \sum_{y_{i}<0} \eta_{i} \\
& =\frac{1}{2} \Delta_{b a}(\bar{h}(\overline{\mathbf{x}}), \bar{y})
\end{aligned}
$$

we see that the right-hand-expression of each inequality constraint in $\mathrm{SVM}_{b a}^{\text {perf }}$ also turns out to be same as that in $\mathrm{SVM}_{b a}^{\text {struct }}$. Hence both optimisation problems would lead to the same solution $\mathrm{w}^{*}$.

Theorem 3. $\mathrm{SVM}_{b a}^{\text {perf }}\left(\mathrm{SVM}_{b a}^{\text {struct }}\right)$ can be trained in linear time w.r.t. the size of $P \cup U$, i.e., $l+u$.

Proof. It has been shown that SVM ${ }^{\text {struct }}$ can be correctly trained by the cutting-plane algorithm in $O(s n)$ time where $s$ is the average number of non-zero features and $n$ is the number of training examples [5]. The same algorithm can be adapted for the training of $\mathrm{SVM}_{b a}^{\text {struct }}$ with little modification. In our case, there are $n=|P \cup U|=l+u$ training examples.

\section{Precision-Recall Product}

In information retrieval applications, performance is more often evaluated in terms of precision and recall [12] rather than accuracy.

The actual precision and recall are

$$
p=\operatorname{Pr}[z=1 \mid h(\mathbf{x})=1] \text { and } r=\operatorname{Pr}[h(\mathbf{x})=1 \mid z=1]
$$

respectively.

The observed precision and recall are

$$
\hat{p}=\operatorname{Pr}[y=1 \mid h(\mathbf{x})=1] \text { and } \hat{r}=\operatorname{Pr}[h(\mathbf{x})=1 \mid y=1]
$$

respectively.

Generally speaking, we want both precision and recall to be high in a retrieval situation. The $F_{1}$ score, which addresses precision and recall equally, is probably the most popular multivariate performance measure in IR [12]. It is defined as the harmonic average of precision and recall,

$$
F_{1}=\frac{2}{\frac{1}{p}+\frac{1}{r}}=\frac{2 p r}{p+r} .
$$

Unfortunately in PU Learning, high observed $F_{1}$ does not guarantee high actual $F_{1}$.

We turn to optimise an alternative multivariate performance measure $-p r$, the product of precision and recall. Similar to the $F_{1}$ score, $p r$ is high when both precision and recall are high, but low when either of them is low. In fact $p r$ correlates with $F_{1}$ closely. It is easy to see that $p r$ is a lower-bound of $F_{1}$ and $\sqrt{p r}$ is an upper-bound of $F_{1}$. 
Theorem 4. $p r \leq F_{1} \leq \sqrt{p r}$.

Proof. $F_{1} \geq p r$ because $0 \leq p+r \leq 2 . \quad F_{1} \leq \sqrt{p r}$ because the harmonic average can never be greater than the geometric average.

The relationship between the observed precision/recall and the actual precision/recall is given by the following two lemmas.

Lemma 1. $\hat{r}=r$.

Proof. This comes directly from the assumption that the examples in $P$ is randomly sampled from the class of actual positive examples.

$$
\begin{aligned}
\hat{r} & =\operatorname{Pr}[h(\mathbf{x})=1 \mid y=1] \\
& =\frac{\operatorname{Pr}[h(\mathbf{x})=1, y=1]}{\operatorname{Pr}[y=1]} \\
& =\frac{\operatorname{Pr}[h(\mathbf{x})=1, z=1] \mu}{\operatorname{Pr}[z=1] \mu} \\
& =\operatorname{Pr}[h(\mathbf{x})=1 \mid z=1] \\
& =r .
\end{aligned}
$$

Lemma 2. $\hat{p} \propto p$.

Proof.

$$
\begin{aligned}
\hat{p} & =\operatorname{Pr}[y=1 \mid h(\mathbf{x})=1] \\
& =\frac{\operatorname{Pr}[h(\mathbf{x})=1 \mid y=1] \operatorname{Pr}[y=1]}{\operatorname{Pr}[h(\mathbf{x})=1]} \\
& =\frac{\hat{r} \operatorname{Pr}[z=1] \mu}{\operatorname{Pr}[h(\mathbf{x})=1]}=\frac{r \operatorname{Pr}[z=1] \mu}{\operatorname{Pr}[h(\mathbf{x})=1]} \\
& =\frac{\operatorname{Pr}[z=1, h(\mathbf{x})=1]}{\operatorname{Pr}[h(\mathbf{x})=1]} \mu \\
& =\operatorname{Pr}[z=1 \mid h(\mathbf{x})=1] \mu \\
& =p \mu \propto p .
\end{aligned}
$$

Theorem 5. $\widehat{p r} \propto p r$.

Proof. It is simply because $\hat{p} \propto p$ and $\hat{r}=r$.

This theorem implies that we can optimise the actual precision-recall product $p r$ by optimising the observed precision-recall product $\widehat{p r}$.

Since $0 \leq \widehat{p r} \leq 1$, we define the corresponding multivariate loss function as

$$
\Delta_{p r}(\bar{h}(\overline{\mathbf{x}}), \bar{y})=1-\widehat{p r} .
$$

Let $\mathrm{SVM}_{p r}^{\text {perf }}$ denote the $\mathrm{SVM}^{\text {perf }}$ with the loss function $\Delta_{p r}$.
Theorem 6. $\mathrm{SVM}_{p r}^{\text {perf }}$ can be trained in polynomial time w.r.t. the size of $P \cup U$, i.e., $l+u$.

Proof. It has been shown that if the loss function $\Delta$ can be computed from the following contingency table, $\mathrm{SVM}^{\text {perf }}$ can be correctly trained by a sparse-approximation algorithm [15] in $O\left(n^{2} t\right)$ time where $n$ is the number of training examples and $t$ is the number of different contingency tables [5].

\begin{tabular}{|c|c|c|}
\hline & $\begin{array}{c}y=+1 \\
(\mathbf{x} \in P)\end{array}$ & $\begin{array}{c}y=-1 \\
(\mathbf{x} \in U)\end{array}$ \\
\hline$h(\mathbf{x})=+1$ & $\mathrm{a}$ & $\mathrm{b}$ \\
$h(\mathbf{x})=-1$ & $\mathrm{c}$ & $\mathrm{d}$ \\
\hline
\end{tabular}

$\Delta_{p r}(\bar{h}(\overline{\mathbf{x}}), \bar{y})$ can be computed from the contingency table:

$$
\Delta_{p r}(\bar{h}(\overline{\mathbf{x}}), \bar{y})=1-\frac{a^{2}}{(a+b)(a+c)} .
$$

Given $P$ and $U$, the values in such a contingency table must satisfy the constraints $a, b, c, d \geq 0, a+c=l$ and $b+d=u$. Although there are $n !=(l+u)$ ! different rankings, there are only $(l+1)(u+1) \in O\left(n^{2}\right)$ different contingency tables that are legitimate. Therefore $\mathrm{SVM}_{p r}^{\text {perf }}$ can be trained in at most $O\left(n^{4}\right)$ time.

\section{Experiments}

\subsection{Code}

We have implemented the proposed SVM learning algorithms on the basis of Joachim's SVM ${ }^{\text {perf } 1}$. Our source code will be made available at the first author's homepage.

\subsection{Data}

We conduct our experiments on the following three realworld datasets which are pre-processed and publicly available $^{2}$.

- The news20 dataset contains approximately 20,000 articles that were collected from 20 different newsgroups.

- The siam-competition2007 dataset contains 28,596 aviation safety reports that were used in the SIAM Text Mining Competition 2007.

- The mediamill-exp1 dataset contains 43,907 camerashots from 85 hours of international news broadcast video data that were used in the MediaMill Challenge Problem for generic video indexing. Only the top 5 semantic concepts are used as categories in our experiments.

\footnotetext{
${ }^{1}$ http://svmlight.joachims.org/svm_perf.html

${ }^{2}$ http://www.csie.ntu.edu.tw/ cjlin/libsvmtools/datasets/
} 


\subsection{Setting}

We construct binary classification tasks based on the default train/test split for each dataset. Given a category, the positive set $P$ consists of the relevant examples before the split point, the negative set $N$ consists of the irrelevant examples before the split point, while the unlabelled set $U$ consists of all (relevant and irrelevant) examples after the split point. When the negative set is available, we can use both $P$ and $N$ to train $\mathrm{SVM}_{2 c}$ which provides the ideal classification performance or the upper-bound for PU Learning. When the negative set is not available, we can use $P$ only to train $\mathrm{SVM}_{1 c}$, or use both $P$ and $U$ to train our proposed $\mathrm{SVM}_{b a}^{\text {perf }}$ and $\mathrm{SVM}_{p r}^{\text {perf }}$.

\subsection{Results}

We evaluate the classification effectiveness of SVMs on each dataset using the average accuracy, $F_{1}$ score and Area Under the ROC Curve (AUC), as shown in Table 1. We see that $\mathrm{SVM}_{b a}^{\text {perf }}$ and $\mathrm{SVM}_{p r}^{\text {perf }}$ work very well in the PU Learning setting on all the datasets: their classification performances are much higher than that of $\mathrm{SVM}_{1 c}^{\text {perf }}$, and are as good as that of $\mathrm{SVM}_{2 c}^{\text {perf }}$ which makes of negative examples. This implies that our proposed classifiers $\mathrm{SVM}_{b a}^{\text {perf }}$ and $\mathrm{SVM}_{p r}^{\text {perf }}$ can be trained effectively no negative examples at all.

We run our experiments on a PC with Pentium $4(3 \mathrm{GHz})$ processor and 2GB memory, and report the average training time (in CPU seconds) of SVMs for PU Learning in Table 2. We see that $\mathrm{SVM}_{b a}^{\text {perf }}$ and $\mathrm{SVM}_{p r}^{\text {perf }}$ both can be trained efficiently. Moreover, $\mathrm{SVM}_{b a}^{\text {perf }}$ runs an order of magnitude faster than $\mathrm{SVM}_{p r}^{\text {perf }}$ : the linear time complexity of $\mathrm{SVM}_{b a}^{\text {perf }}$ makes it more scalable than $\mathrm{SVM}_{p r}^{\text {perf }}$.

\section{Table 2. The efficiency (training time) of SVMs for PU Learning.}

\begin{tabular}{|l|r|r|}
\hline \hline dataset & SVM $_{b a}^{\text {perf }}$ & SVM $_{p r}^{\text {perf }}$ \\
\hline \hline news20 & 0.8905 & 7.1325 \\
\hline siam-competition2007 & 1.0409 & 43.0641 \\
\hline mediamill-exp1 & 3.6140 & 731.3120 \\
\hline \hline
\end{tabular}

\section{Related Work}

The problem of PU Learning has attracted much attention from information retrieval and data mining researchers in recent years $[3,2,7,11,10,8,4,18,17,19,20]$. Please refer to Liu's new book on Web data mining [9] for a comprehensive survey of this field.
Generally speaking, most existing approaches to PU Learning follow a two-step heuristic: (1) constructing a small reliable negative set $\widehat{N}$ by extracting some examples from $U$ which look very unlike positive examples; (2) building a classifier based on $P$ and $\widehat{N}$ iteratively. Our reduction approach to PU Learning is fundamentally different with them.

The work most related to ours is probably the BiasedSVM method which has shown excellent classification performance in comparison to other state-of-the-art PU Learning methods [10]. It also attempts to optimise a multivariate performance measure (proportional to $p r$ ), but through an indirect trail-and-error way: it tries a large number (typically hundreds) of SVMs each with a different cost parameter and then pick one from them according to the classification performance on a held-out validation set. So theoretically the classification performance of Biased-SVM could not be better than $\mathrm{SVM}_{p r}^{\text {perf }}$. Our reduction approach to PU Learning has several advantages over Biased-SVM:

- it should be more effective because it directly optimises meaningful multivariate performance measures;

- it is hundreds of times more efficient because it only needs to train one classifier;

- a held-out validation set is no longer a prerequisite.

\section{Conclusions}

In this paper, we have proposed to solve the problem of PU Learning by reducing it to the problem of learning classifiers for some meaningful multivariate performance measures (namely balanced accuracy and precision-recall product). Specifically we have presented two variants of standard SVM, SVM $p r$ perf and SVM $p r f$, which can be used for effective and efficient PU Learning.

\section{Acknowledgements}

We thank the anonymous reviewers for their helpful comments.

\section{References}

[1] A. Blum and T. Mitchell. Combining labeled and unlabeled data with co-training. In Proceedings of the 11th Annual Conference on Computational Learning Theory (COLT), pages 92-100, Madison, WI, 1998.

[2] F. Denis, R. Gilleron, and F. Letouzey. Learning from positive and unlabeled examples. Theoretical Computer Science, 348(1):70-83, 2005. 
Table 1. The effectiveness of SVMs for PU Learning.

\begin{tabular}{|l|l|c|cc|c|}
\hline \hline dataset & measure & $\mathrm{SVM}_{1 c}$ & $\mathrm{SVM}_{b a}^{\text {erf }}$ & $\mathrm{SVM}_{p r}^{\text {perf }}$ & $\mathrm{SVM}_{2 c}$ \\
\hline \hline \multirow{3}{*}{ 20news } & accuracy & 0.7771 & 0.9795 & 0.9802 & 0.9805 \\
\cline { 2 - 6 } & $F_{1}$ & 0.2751 & 0.7771 & 0.7887 & 0.7538 \\
\cline { 2 - 6 } & ROC-AUC & 0.9116 & 0.9877 & 0.9881 & 0.9904 \\
\hline \hline \multirow{3}{*}{ siam-competition2007 } & accuracy & 0.4072 & 0.9389 & 0.9267 & 0.9448 \\
\cline { 2 - 6 } & $F_{1}$ & 0.1774 & 0.5499 & 0.5606 & 0.4251 \\
\cline { 2 - 6 } & ROC-AUC & 0.9009 & 0.9740 & 0.9736 & 0.9624 \\
\hline \hline \multirow{3}{*}{ mediamill-exp1 } & accuracy & 0.4517 & 0.7823 & 0.6640 & 0.7965 \\
\cline { 2 - 6 } & $F_{1}$ & 0.5841 & 0.5950 & 0.6501 & 0.5846 \\
\cline { 2 - 6 } & ROC-AUC & 0.7732 & 0.8850 & 0.8833 & 0.8961 \\
\hline \hline
\end{tabular}

[3] F. Denis, R. Gilleron, and M. Tommasi. Text classification from positive and unlabeled examples. In Proceedings of the 9th International Conference on Information Processing and Management of Uncertainty in Knowledge-Based Systems (IPMU), pages 1927-1934, Annecy, France, 2002.

[4] G. P. C. Fung, J. X. Yu, H. Lu, and P. S. Yu. Text classification without negative examples revisit. IEEE Transaction of Knowledge and Data Engineering (TKDE), 18(1):6-20, 2006.

[5] T. Joachims. A support vector method for multivariate performance measures. In Proceedings of the 22nd International Conference on Machine Learning (ICML), pages 377-384, Bonn, Germany, 2005.

[6] T. Joachims. Training linear SVMs in linear time. In Proceedings of the 12th ACM SIGKDD International Conference on Knowledge Discovery and Data Mining (KDD), pages 217-226, Philadelphia, PA, 2006.

[7] W. S. Lee and B. Liu. Learning with positive and unlabeled examples using weighted logistic regression. In Proceedings of the 20th International Conference on Machine Learning (ICML), pages 448-455, Washington, DC, 2003.

[8] X. Li and B. Liu. Learning to classify texts using positive and unlabeled data. In Proceedings of the 18th International Joint Conference on Artificial Intelligence (IJCAI), pages 587-594, Acapulco, Mexico, 2003.

[9] B. Liu. Web Data Mining: Exploring Hyperlinks, Contents and Usage Data. Springer, 2006.

[10] B. Liu, Y. Dai, X. Li, W. S. Lee, and P. S. Yu. Building text classifiers using positive and unlabeled examples. In Proceedings of the 3rd IEEE International Conference on Data Mining (ICDM), pages 179-188, Melbourne, FL, 2003.

[11] B. Liu, W. S. Lee, P. S. Yu, and X. Li. Partially supervised classification of text documents. In Proceedings of the 19th International Conference (ICML), pages 387-394, Sydney, Australia, 2002.

[12] C. D. Manning, P. Raghavan, and H. Schütze. Introduction to Information Retrieval. Cambridge University Press, 2008.

[13] B. Scholkopf and A. J. Smola. Learning with Kernels. MIT Press, Cambridge, MA, 2002.

[14] M. Sokolova, N. Japkowicz, and S. Szpakowicz. Beyond accuracy, F-score and ROC: a family of discriminant measures for performance evaluation. In Proceedings of the AAAI'06 workshop on Evaluation Methods for Machine Learning, 2006.

[15] I. Tsochantaridis, T. Joachims, T. Hofmann, and Y. Altun. Large margin methods for structured and interdependent output variables. Journal of Machine Learning Research (JMLR), 6:1453-1484, Sep 2005.

[16] Y. Yang and X. Liu. A re-examination of text categorization methods. In Proceedings of the 22nd Annual International ACM SIGIR Conference on Research and Development in Information Retrieval (SIGIR), pages 42-49, Berkeley, CA, 1999.

[17] H. Yu. Single-class classification with mapping convergence. Machine Learning, 75(1):49-69, 2005.

[18] H. Yu, J. Han, and K. C.-C. Chang. PEBL: Web page classification without negative examples. IEEE Transactions on Knowledge and Data Engineering (TKDE), 16(1):70-81, 2004.

[19] D. Zhang and W. S. Lee. A simple probabilistic approach to learning from positive and unlabeled examples. In Proceedings of the 5th Annual UK Workshop on Computational Intelligence (UKCI), pages 83-87, London, UK, 2005.

[20] D. Zhang and W. S. Lee. Learning with support vector machines for query-by-multiple-examples. In Proceedings of the 31st Annual International ACM SIGIR Conference on Research and Development in Information Retrieval (SIGIR), pages 835-836, Singapore, 2008. 